Supplementary material

\title{
Homeopathic treatment of two patients with coronary artery disease: case-report
}

Pedro Bernardo Scala

Universidad Maimonides, Buenos Aires, Argentina

Main article: Scala PB. Homeopathic treatment of two patients with coronary artery disease: case-report. Int J High Dilution Res [online]. 2009 [cited 2009 Jun 30]; 8 (27): 4549. Available from: http://www.feg.unesp.br/ ojs/index.php/ijhdr/article/view/337/391.

\section{INSTITUTO CARDIOVASCULAR DE BUENOS AIRES SERVICIO DE CARDIOLOGÍA INTERVENCIONISTA Y TERAPEUTICAS ENDOVASCULARES}

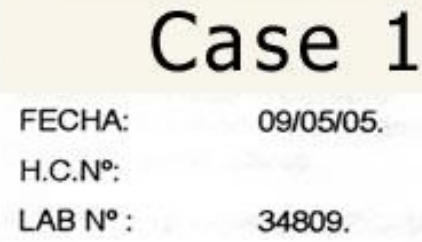




Sardiovex-ergometrit
Fagina 1

Estudio Numero: 2647 Veronesi Anita 23-06-2004

TTOS PERSONALES

\section{Case 1}

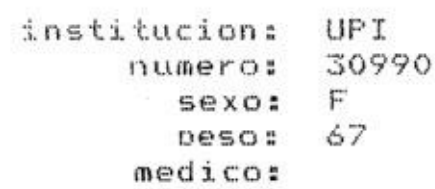

H.C.:

edad: 65

al tura: 1.65

\section{ITECEDENTES}

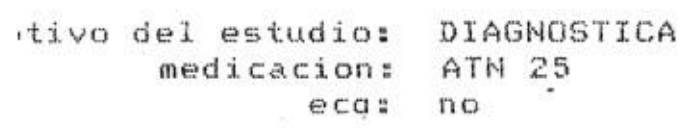

MCLUSTOHES

PRUEBA DETENTDA FOF AGOT. MUSCULAF A LOS A LOS 450 KGIS. REFTERE ANGOR*/4 QUE CEDE CON LA RECUF INTEDIATA " RECTIFTCACION DEL. ST INTRAESFUERZO EN V5 COM THFRAST DE DE O. 5 MIV EN VS E TNUERSION DE LAA ONDA $T$ TANTO EN VS COMO EN V2. EN EL. FOSTESFUEFZO.

PEG POSITIVA PARA TSQUEMIA. 


\section{CINECORONARIOGRAFIA}

\section{Tronco de Coronaria Izquierdo}

Con irregularidades, sin lesiones significativas.

\section{Arteria Descendente Anterior}

De gran calibre y tamaño, la cual irriga hasta el ápex cardiaco, sin lesiones significativas.

\section{Arteria Circunfleja}

Con gran tortuosidad la cual brinda un gran ramo LV con irregularidades sin lesiones significativas. Resto de la arteria sin lesiones significativas.

\section{Arteria Coronaria Derecha}

De moderado calibre y extensión, con obstrucción de un $30-40 \%$ en su tercio medio, sin lesiones significativas.

\section{VENTRICULOGRAMA IZQUIERDO}

Ventrículo Izquierdo en OAD.

No dilatado.

Hipertrófico.

Motilidad parietal conservada.

FSVI conservada.

\section{PRESIONES Y OXIMETRIAS}

\begin{tabular}{|l|l|l|l|}
\hline & FASICA & MEDIA & $\mathrm{O}_{2}$ \\
\hline VCI & & & \\
\hline VCS & & & \\
\hline AD & & & \\
\hline VD & & & \\
\hline AP & & & \\
\hline CAP & & & \\
\hline A & & & \\
\hline VI & $160-15$ & & \\
\hline AORTA & $160-70$ & & \\
\hline
\end{tabular}

CONCLUSIONES:

* Arterias coronarias sin lesiones significativas.

Dr. Jor

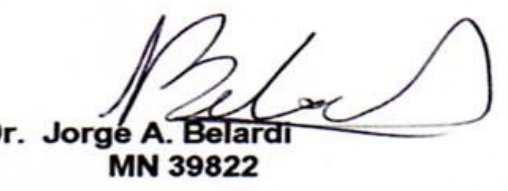

\section{(c)) BY-NC-ND Licensed to GIRI}

Support: authors declare that this study received no funding

Conflict of interest: authors declare there is no conflict of interest

Received: 30 Apr 2009; Revised 24 Jun 2009; Published: 30 Jun 2009

Correspondence author: Pedro Bernardo Scala, pedroscala@gmail.com

How to cite this article: Scala PB. Homeopathic treatment of two patients with coronary artery disease: case-report [Supplementary material]. Int J High Dilution Res [online]. 2009 [cited YYYY Month dd]; 8 (27 Suppl 1): S1-S3. Available from: http://www.feg.unesp.br/ ojs/index.php/ijhdr/article/view/337/389. 